\title{
Early career investigator highlight: May
}

\author{
Wissam Shalish ${ }^{1}$
}

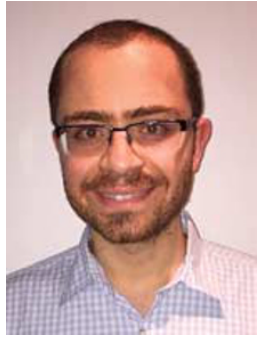

grew up in Montreal and began my medical journey in

2004 at McGill University. I had always been attracted to Pediatrics, but it was only in the latter years of medical school that my passion was concretized; I particularly loved the unique dual interactions that I encountered with both patients and their parents. At the time, I also took part in a small study involving extremely preterm infants under the supervision of Dr Keith Barrington. This serendipitous research opportunity was an exceptional experience that became the catalyst for my interest in pursuing a career in Neonatology and Clinical Research.

After graduating from medical school, I started residency in Pediatrics at the Montreal Children's Hospital. During training, I met Dr Therese Perreault and Dr Guilherme Sant'Anna, two neonatologists who rapidly became major influences in my career path. With their precious guidance, I pursued Neonatal fellowship at the Hospital for Sick Children in Toronto, where I was trained by truly inspiring colleagues.
In 2013, I returned to Montreal as Neonatologist at the Montreal Children's Hospital. With the desire to build a solid foundation in clinical research, I was given the possibility to enroll in the $\mathrm{PhD}$ program in Experimental Medicine under the supervision and remarkable mentorship of Dr Sant'Anna. My thesis has focused on understanding the magnitude, clinical implications, and predictors of extubation failure in extremely preterm infants. I have been fortunate to be at the forefront of a large multicenter prospective study looking at the use of automated analyses of cardiorespiratory signals to predict extubation readiness in extremely preterm infants. Through multidisciplinary collaboration with clinicians, respiratory physiologists, biomedical engineers, and computer scientists, I acquired some unique skills in respiratory physiology and biomedical signal analysis, the results of which have led to the work highlighted in this issue.

The PhD has been a steep yet thoroughly rewarding and exciting journey, which has provided me with the necessary tools to establish myself as an independent investigator. Moving forward, I hope to use these newfound skills to better understand cardiorespiratory behavior in extremely preterm infants, with the ultimate goal of providing more individualized and evidence-based respiratory care to this vulnerable population. My training has also taught me a great deal about the importance of discipline in research: stay focused, be patient, and meticulous. Finally, I learnt the value of fostering partnerships, and most importantly, finding mentors who can provide guidance along the way. 